\title{
LAND USE AND LAND COVER CLASSIFICATION OF MULTISPECTRAL LANDSAT-8 SATELLITE IMAGERY USING DISCRETE WAVELET TRANSFORM
}

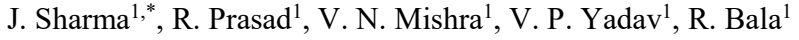 \\ Department of Physics, Indian Institute of Technology (BHU), Varanasi - (jyotisharma.rs.phy17, rprasad.app, \\ vnmishra.rs.app13)@itbhu.ac.in, (victory327759, ruchibala7)@gmail.com
}

Commission V, SS: Emerging Trends in Remote Sensing

KEYWORDS: LULC, LANDSAT-8, DWT, Minimum distance classifier, Kappa coefficient

\begin{abstract}
:
Land use and land cover (LULC) classification of satellite imagery is an important research area and studied exclusively in remote sensing. However, accurate and appropriate land use/cover detection is still a challenge. This paper presents a wavelet transform based LULC classification using Landsat 8-OLI data. The study area for the present work is a small part of Varanasi district, Uttar Pradesh, India. The atmospheric correction of the image was performed using Quick Atmospheric Correction (QUAC) method. The image was decomposed into its approximation and detail coefficients up to eight levels using discrete wavelet transform (DWT) method. The approximation images were layer stacked with the original image. The minimum distance classifier was used for classifying the image into six LULC classes namely water, agriculture, urban, fallow land, sand, and vegetation. The classification accuracy for all decomposition levels was compared with that of classified product based on original multispectral image. The classification accuracy for multi-spectral image was found to be $75.27 \%$. Whereas, the classification accuracies were found to improve up to $85.97 \%, 88.87 \%, 93.47 \%, 95.03 \%, 93.01,92.32 \%$ and $90.80 \%$ for second, third, fourth, fifth, six, seventh and eight level decomposition, respectively. The significantly improved accuracy was found for images decomposed at level five. Thus, the approach of DWT for LULC classification can be used to increase the classification accuracy significantly.
\end{abstract}

\section{INTRODUCTION}

LULC classification plays a noteworthy role in development of region and nation. Accurate and appropriate information of LULC is extremely important for analyzing various socio-ecological concerns. This information is vital in several applications including urban land planning, agriculture, rural management and sustainable development. Remote sensing technology is extensively used to study the estimation of LULC changes. Remote sensing data obtained from many satellites have been broadly used to retrieve LULC information (Babykalpana et al., 2010; Saadat et al., 2011). There are a variety of methods have been introduced for LULC classification with the development and advances in remote sensing technology and satellites (Sekertekin et al., 2017; Mishra et al., 2014). In this sequence, Landsat -8 OLI is recently launched earth observational satellite. Many techniques have been introduced to retrieve LULC image from Landsat-8 OLI data to investigate better classification accuracy (Phiri et al., 2017; Jia et al., 2014; Taufik et al., 2016). Several DWT based methods have been developed for LULC classification of many satellites (Chen et al., 2007; Rajesh et al., 2011; Myint et al., 2006). In the present study, DWT approach has been used on Landsat -8 OLI data for LULC classification. The DWT is applied on the image to decompose it into its different frequency components. These frequency components are known as wavelet coefficients.

Thus, the purpose of the paper is to use different decomposition level of DWT (from second to eight) on Landsat- 8 images followed by using Minimum Distance (MDC) supervised classification technique to generate LULC image to reveal that which decomposition level gives better accuracy.

\section{STUDY AREA}

Varanasi district, located in Uttar Pradesh (East), north India is selected as the study area. Varanasi is the oldest city of the India and one of the oldest cities of the world. Another name of Varanasi is Kashi situated at the bank of divine river Ganga, the longest river of India. The land is very fertile because of its location in Indo - Gangetic plain. The area covered by it is $144 \mathrm{~km}^{2}$ having center latitude $25^{\circ} 15^{\prime} 55.27^{\prime \prime} \mathrm{N}$ and longitude $82^{\circ} 58^{\prime} 56.92^{\prime \prime} \mathrm{E}$.

Landsat -8 OLI data, acquired on $20 / 11 / 2017$, was used as satellite imagery in this study. Landsat- 8 images contain eleven spectral bands. All these bands having spatial resolution of 30 meters except band 8 and that is 15 meters. Figure 1 shows false color composite (FCC) image of

\footnotetext{
${ }^{*}$ Corresponding author
} 
Landsat- 8 used for this study with the location of study area.

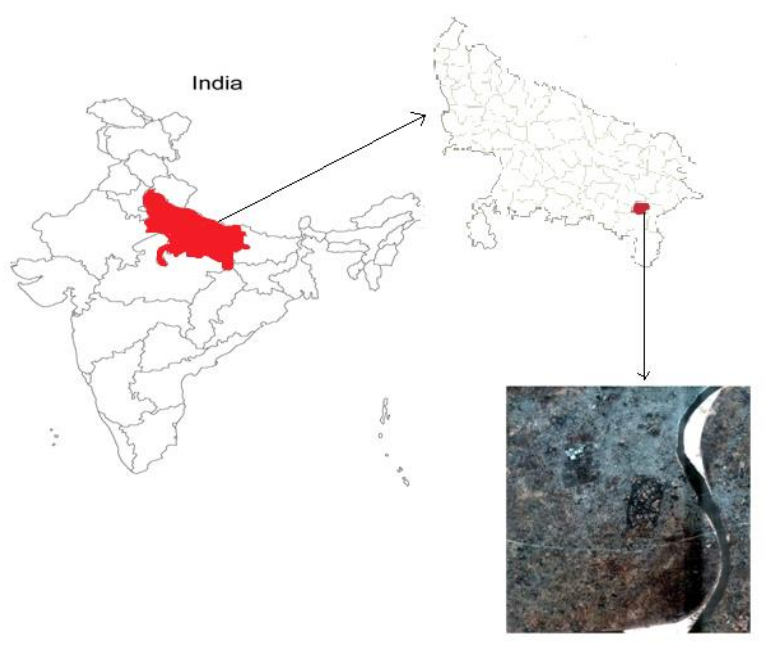

Figure1.Study area (Varanasi, Uttar Pradesh, India) with the FCC image of Landsat -8

\section{METHODOLOGY}

Before classifying, the image was pre-processed in ENVI 5.1 software. The image was atmospheric corrected using QUAC method. The DWT transformed the image from spatial to frequency components.

\subsection{Discrete Wavelet Transform}

DWT decomposed a signal into its different frequency component. The component of low frequency is named as approximation and of high frequency is named as detailed component. These components are known as wavelet coefficients. In the similar manner, DWT can also be used on an image to decompose it. Different frequency constituents of the image obtained after decomposition are approximation (low frequency area of the image) and detailed component (high frequency area of the image) (Gonzalez et al., 1992; Gupta et al., 2015).

The wavelet coefficients are acquired by stretching and translating the mother wavelet. There are various mother wavelets used in DWT such as Coiflet, Morlet, Daubechies wavelet, Biorthogonal, Haar wavelet etc (Graps et al., 1995). In the present study, DWT with Haar wavelet is used for decomposing the image. The mother wavelet function for Haar wavelet (Cheng et al., 2011) can be expressed below

$$
\psi(t)=\left\{\begin{array}{rc}
1, & 0 \leq t<\frac{1}{2} \\
-1, & \frac{1}{2} \leq t<1 \\
0, & \text { otherwise. }
\end{array}\right.
$$

And

$$
\psi_{a, b}(t)=2^{a} / 2 \psi\left(2^{a} t-b\right)
$$

Here, ' $a$ ' is always a nonnegative integer whereas $\mathrm{b}$ lies in the range of $0 \leq \mathrm{b} \leq 2^{a}-1$. The Haar wavelet is shown in Figure 2.

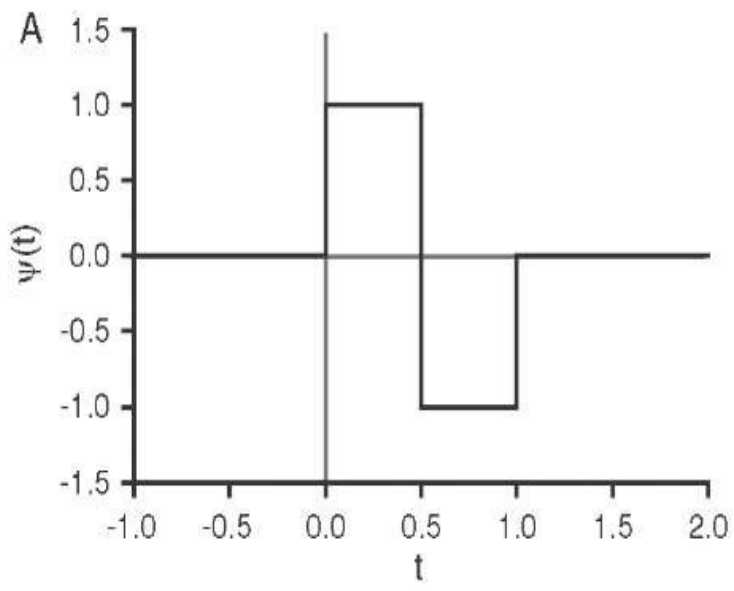

Figure2.The Haar wavelet

The approximation image obtained after decomposition was again subjected to DWT for second time decomposition which is acknowledged as second level decomposition. Similarly for third, fourth and so on up to eight times decomposition can be defined. The layer stacked product of approximation image with the two bands of original Landsat- 8 images gives the combined information of different frequencies.

\subsection{Minimum Distance Classifier}

There are many supervised classification methods for examples MDC, Maximum likelihood classifier, neural network, Mahalanobis distance, Support vector machine etc. If training samples for classes are limited then an algorithm is needed to use only mean value for the classes. MDC or minimum distance to class is an approach where class mean is evaluated by using training samples and then consign pixels to the class of nearest mean (Lillesand et al., 2004). That's why here MDC is used for LULC classification. Six most important LULC classes including water, agriculture, urban, fallow land, sand, and vegetation were chosen for this study. 


\subsection{Accuracy assessment}

The classification accuracy can be defined as the number of pixels which are correctly classified to the sum of all pixels. It is predicted by evaluating overall accuracy along with kappa coefficient. These values are calculated by using confusion matrix (Lillesand et al., 2004). The kappa coefficient can be estimated by using an equation defined below,

$$
\kappa=\frac{N \sum_{i=1}^{r} x_{i i}-\sum_{i=1}^{r} x_{i+} \cdot x_{+i}}{N^{2}-\sum_{i=1}^{r} x_{i+} \cdot x_{i+}}
$$

Where

$\mathrm{N}=$ All number of pixels used in the matrix,

$\mathrm{r}=$ Number of classes or rows or columns in the matrix,

$x_{i i}=$ Value of diagonal elements in the matrix or correctly classified pixels,

$x_{i+}=$ sum of pixels described in $\mathrm{i}^{\text {th }}$ row,

$x_{+i}=$ sum of pixels described in $i^{\text {th }}$ column.

\section{RESULTS}

Landsat-8 images were used for LULC classification using MDC supervised classification method preceded by DWT decomposition. The layer stacked product of approximation image obtained by decomposition at every level and original image was classified by MDC. Figure 3 shows the classified image of the original multispectral image and for the decomposition at level five. The classification accuracy is estimated for original classified image and for every decomposition level. The original classified image gives overall accuracy of $75.2693 \%$ with the kappa coefficient 0.6827 . Table 1 shows the estimated accuracy for each level which indicates that the classification accuracy is first increases from second to fifth level and then starts decreasing up to eight levels. Thus the classified image for decomposition level-five gives the maximum classification accuracy.

\begin{tabular}{|l|l|l|}
\hline $\begin{array}{l}\text { Wavelet } \\
\text { decomposition } \\
\text { level }\end{array}$ & $\begin{array}{l}\text { Overall } \\
\text { accuracy (\%) }\end{array}$ & $\begin{array}{l}\text { Kappa } \\
\text { coefficients }\end{array}$ \\
\hline 2 & 85.96 & 0.8241 \\
\hline 3 & 88.8737 & 0.8622 \\
\hline 4 & 93.4666 & 0.9146 \\
\hline 5 & 95.0346 & 0.9370 \\
\hline 6 & 93.0087 & 0.9110 \\
\hline 7 & 92.3187 & 0.9038 \\
\hline 8 & 90.8021 & 0.8844 \\
\hline
\end{tabular}

Table1.Estimated overall accuracy along with kappa coefficient for classified image at different level of decomposition

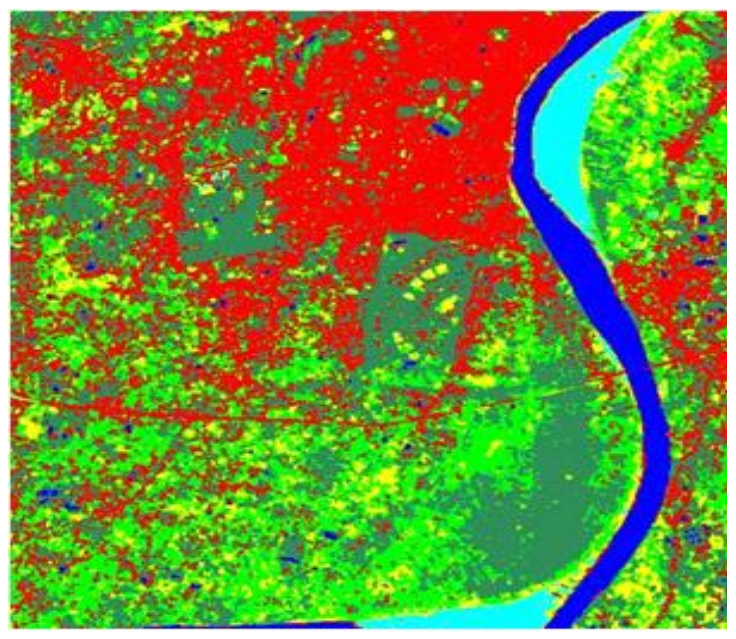

(a)

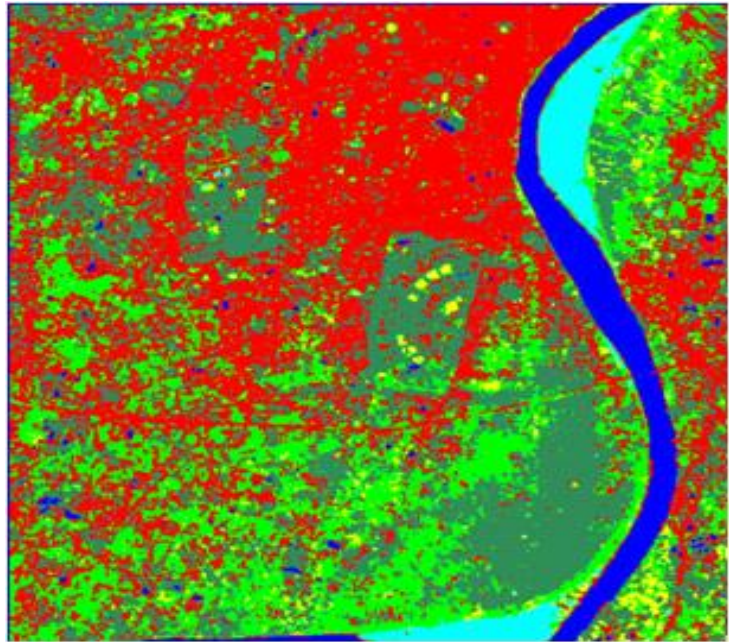

(b)

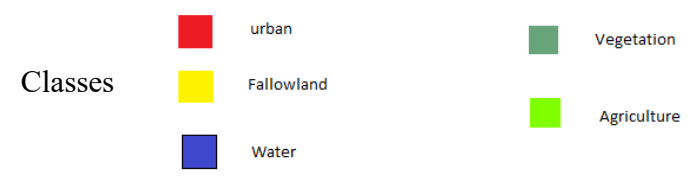

Figure3.Classified image (a) original (b) after decomposition at level-5

\section{CONCLUSIONS}

The consequence of DWT approach on the LULC classification of Landsat-8 OLI imagery was studied in this paper. The classification accuracy was also affected by DWT and found different for different decomposition level. This consequence shows that the classification accuracy is found to be improved if DWT at various level of decomposition (from second to eight) was used before the classifier. It is found maximum for the decomposition at level five significantly. Thus it is concluded that DWT at decomposition level five gives better accuracy. 


\section{ACKNOWLEDGEMENT}

The author is very thankful to the United States Geological Survey (USGS) for providing Landsat - 8 OLI data.

\section{REFERENCES}

Babykalpana, Y., Dr. ThanushKodi, K., 2010. Supervised/Unsupervised Classification of LULC using remotely Sensed Data for Coimbatore city, India. International Journal of Computer Applications, 2 (No.7), pp. $26-30$.

Chen, J., Chen, D., Blostein, D., 2007. Wavelet-Based Classification of Remotely Sensed Images: A Comparative Study of Different Feature Sets in an Urban Environment. Journal of Environmental Informatics, 10(1), pp. 2-9.

Cheng, D. C., 2011. Haar Wavelet Analysis.

Gonzalez, R. C., Woods, R. E., 1992. Digital Image Processing, second edition, New Jersey, pp. $386-389$.

Graps, A., 1995. An Introduction to Wavelets. IEEE COMPUTATIONAL SCIENCE \& ENGINEERING, 2(No. 2), pp. $50-61$.

Gupta, D., Choubey, S., 2015. Discrete Wavelet Transform for Image Processing. International Journal of Emerging Technology and Advanced Engineering, 4, pp. 598 - 602.

Jia, K., Wei, X., Gu, X., Yao, Y., Xie, X., Li, B., 2014. Land cover classification using Landsat 8 Operational Land Imager data in Beijing, China. Geocarto International, 29(No. 8), pp. $941-951$.

Lillesand, T. M., Kiefer, R. W., Chipman, J. W., 2004. Remote sensing and image interpretation, Fifth Edition. WILEY, New Jersey, pp. 556 - 557, pp. $586-591$.

Mishra, V. N., Kumar, P., Gupta, D. K., Prasad, R., 2014. Classification of various land features using risat-1 dual polarimetric data. The International Archives of the Photogrammetry, Remote Sensing and Spatial Information Sciences, XL-8, pp. $833-837$.

Myint, S. W., 2006. A New Framework for Effective Urban Land Use and Land Cover Classification: A Wavelet Approach. GIScience \& Remote Sensing, 43:2, pp. 155178.

Phiri, D., Morgenroth, J., 2017. Developments in Landsat Land Cover Classification Methods: A Review. Remote sensing, 9.

Rajesh, S., Arivazhagan, S., Moses, K. P., Abisekaraj, R., 2011. Land Cover/Land use Mapping Using Different Wavelet Packet Transforms for LISS IV Madurai Imagery. Indian Society of Remote Sensing, 40 (2), pp. 313 - 324.
Saadat, H., Adamowski, J., Bonnell, R., Sharifi, F., Namdar, M., Ale-Ebrahim, S., 2011. Land use and land cover classification over a large area in Iran based on single date analysis of satellite imagery. ISPRS Journal of Photogrammetry and Remote Sensing, 66, pp. $608-619$.

Sekertekin, A., Marangoz, A. M., Akcin, H., 2017. Pixelbased classification analysis of land use land cover using sentinel-2 and landsat- 8 data. The International Archives of the Photogrammetry, Remote Sensing and Spatial Information Sciences, XLII-4/W6, pp. 91 - 93.

Taufik, A., Ahmad, S. S. S., 2016. Land cover classification of Landsat 8 satellite data based on Fuzzy Logic approach. IOP Conference Series: Earth and Environmental Science, 37. 